\title{
DEVELOPMENT OF CUBE TEXTURE IN COARSE GRAINED COPPER
}

\author{
M. SINDEL,${ }^{1} \dagger$ G. D. KÖHLHOFF, ${ }^{1}$ K. LÜCKE ${ }^{1}$ and B. J. DUGGAN ${ }^{2}$
}

\author{
${ }^{1}$ Institut für Allgemeine Metallkunde und Metallphysik der RWTH Aachen, \\ Kopernikusstraße 14, D-5100 Aachen, F.R.G. \\ ${ }^{2}$ Department of Mechanical Engineering, University of Hong Kong, \\ Pokfulam Road, Hong Kong
}

(Received January 18, 1989; in final form February 3, 1989)

Cube oriented deformed material has been detected in the rolling texture measured on edge sections of heavily rolled coarse grained copper. The level of intensity is low, and seems to be independent of rolling reduction over the range 93-98\%. Recrystallization textures show an increasing strength of Cube with rolling reduction. The evidence is consistent with the idea that Cube nuclei are created by a mechanism similar to that proposed by Dillamore and Katoh, and the strength of the recrystallized Cube texture depends on oriented growth. Prior grain size effects are briefly examined and it is shown that deformation textures are less sharp in large grain size compared with small grain sized copper at similar strains. It is likely that the effect of grain size on cube texture formation arises from grain size dependent texture changes in the vicinity of the deformed Cube oriented material.

KEY WORDS Cube texture, copper, grainsize, nucleation, growth selection.

\section{INTRODUCTION}

The development of the Orientation Distribution Function (ODF) technique has allowed FCC rolling textures to be described in terms of fibres. In intermediate to high Stacking Fault Energy (SFE) metals and alloys which form the copper-type texture at high rolling reductions these fibres are called $\alpha$ and $\beta$ (Hirsch and Lücke, 1988). The $\alpha$ fibre can be visualized as a rotation of $\pm 35^{\circ}$ from the orientation $\{110\}\langle 001\rangle$ about ND and the $\beta$ fibre by a rotation of the orientation $\{112\}\langle 111\rangle$ by approximately $\pm 35^{\circ}$ about the $\langle 110\rangle$ pole which is located in the plane described by ND/RD and inclined $\pm 35^{\circ}$ to RD. Along the $\alpha$ fibre are the orientations Goss ("G") $\{110\}\langle 001\rangle$ and Brass ("B") $\{110\}\langle 112\rangle$ and along the $\beta$ fibre are Copper ("C") $\{112\}\langle 111\rangle$, the orientation $\{123\}\langle 634\rangle$ designated "S" and also Brass ("B"). The copper-type rolling texture forms the well-known Cube texture ("W") $\{001\}\langle 100\rangle$ on annealing providing other conditions such as purity, rolling temperature and degree of deformation are sufficient.

Another factor which is known to affect the development of Cube texture in copper is grain size. It is well established that when the starting grain size is relatively large in copper, the development of Cube texture after rolling and annealing is impaired (Dahl and Pawlek, 1936; Wassermann and Grewen, 1962;

† Now at Technische Universitat Hamburg-Harburg, Harburger Schloßstraße 20, D-2100 Hamburg 90, F.R.G. 
Dillamore and Roberts, 1965). Another observation which seems well founded in the literature, is that the annealed grain size in copper which has a well developed Cube texture, is always relatively large. Both of these grain size observations have been discussed and plausible explanations offered by Ridha and Hutchinson (1982) and Hutchinson and Nes (1986). The origin of Cube texture has been ascribed to oriented nucleation by many authors, and the most developed model is due to Dillamore and Katoh (1974). Alternatively its origin, in terms of the modern oriented growth theory, is due to Schmidt and Lücke (1979) and Lücke (1984). It is the purpose of this paper to examine again the formation of Cube texture in coarse grain sized copper using pole figure data and ODFs derived from a high accuracy X-ray goniometer and software system developed at Aachen (Hirsch et al., 1986).

\section{EXPERIMENTAL PROCEDURE}

Plates of $99.995 \% \mathrm{Cu}$ were prepared to have a thickness of $32.5 \mathrm{~mm}$ and $17 \mathrm{~mm}$ and grain sizes of $500 \mu \mathrm{m}$ and $50 \mu \mathrm{m}$, respectively. The textures from both plates were weak. They were rolled homogeneously $\left(l_{c} / d \geq 1\right.$, Asbeck and Mecking 1978) on a 2 high mill to 93-98\% reductions using paraffin lubricant and cooling between each pass. Annealing was carried out in an oil bath in the range $140^{\circ} \mathrm{C}-300^{\circ} \mathrm{C}$. (111), (200), (220), and (113) pole figures were measured from each sample using the back-reflection technique, and ODFs were calculated applying the correction techniques developed by Lücke et al., (1981). For part of the work it was necessary to measure pole figures from the longitudinal section of the sheet which was accomplished by glueing strips together in such a way as to retain the rolling/transverse/normal directions of the rolling geometry (package samples). In these cases the ODFs have been rotated mathematically by $90^{\circ}$ in order to obtain the usual representation. The pole figures, in contrast, have been left with the projection plane parallel to RD and ND. Specimens were ground and etched prior to X-ray texture measurement. The $95 \%$ rolled $50 \mu \mathrm{m}$ material which is the most heavily investigated material, was used as a "control" material, i.e. its behaviour was the norm to which the $500 \mu \mathrm{m}$ material was compared.

\section{RESULTS}

\subsection{Rolling Textures}

Ordinary pole figures derived from the sheet surface of $50 \mu \mathrm{m}$ grain sized copper after rolling $95 \%$ and the corresponding true ODF show the typical "coppertype" rolling texture. The texture from the $500 \mu \mathrm{m}$ grain sized copper, however, were unsymmetrical, but symmetry improved as strain increased from $93 \%$ to 98\%. Now it is known from Transmission Electron Microscopy (TEM) (Ridha and Hutchinson, 1982), that Cube oriented volumes are plate-like and lie parallel to the rolling plane which might not favour their quantitative determination from pole figures taken from the sheet surface. This was found to be the case here: (200) intensity was extremely variable, ranging from zero to $0.4 \%(R=$ intensity 
for random pole density distribution). These results are omitted for the sake of brevity. Therefore, in an effort to systematically detect Cube oriented volumes and to increase the sampling statistics, pole figures were measured from the plane parallel to RD and ND (i.e. the longitudinal section, LS). (200) pole figures for this geometry are shown in Figure 1 for $50 \mu \mathrm{m}$ and $500 \mu \mathrm{m}$ material. What is immediately obvious is that Cube-oriented material can be seen at a low level in all of the roller copper. Considerable texture sharpening is evident in Figures $1 \mathrm{~b}-\mathrm{d}$ as rolling strain increased. Also the usual copper-type rolling components are strengthening, and the Cube orientation develops into an incomplete $\{h k l\}\langle 100\rangle$ fibre. It is also true that the LS pole figures are more symmetrical compared with those derived from the sheet surfaces.

True ODFs were calculated from the pole figures derived from the sheet surface and longitudinal sections and intensity variations along the $\beta$ fibre were deduced. Figures $2 a, b$ give examples for ODFs derived from longitudinal sections. The results obtained from the data derived from the sheet surfaces, especially in the larger grain sized material, again showed significant unsystematic differences and, more importantly, did not show the well established behaviour measured by other authors (Hirsch and Lücke, 1988). $\beta$ fibres obtained from the data derived from longitudinal sections, however, are well in agreement with the reported behaviour as can be seen in Figure 3 for the $96 \%$ and $98 \%$
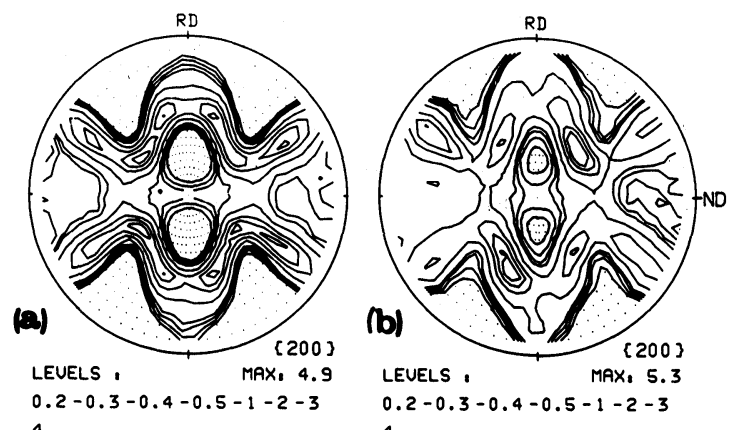

$0.2-0.3-0.4-0.5-1-2-3$

4
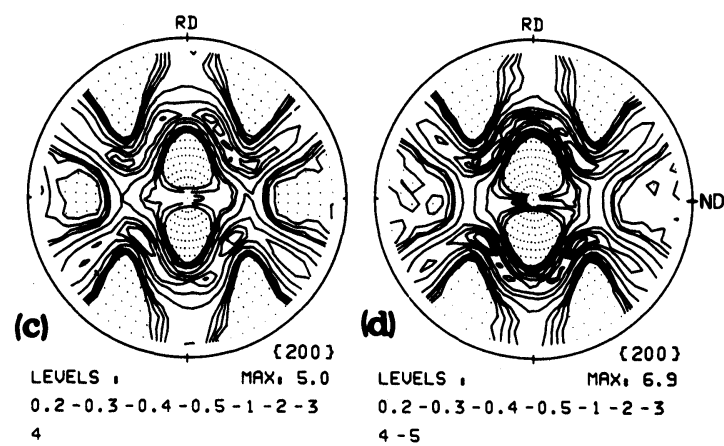

Figure 1 (200) pole figures of cold rolled copper. (a) $50 \mu \mathrm{m}$, cold rolled $95 \%$. (b), (c), and (d) are all from $500 \mu \mathrm{m}$ grain sized material, after rolling $93 \%, 96 \%$, and $98 \%$, respectively. The data was derived from longitudinal sections (Please note change of axis with respect to the usual way of plotting.) 

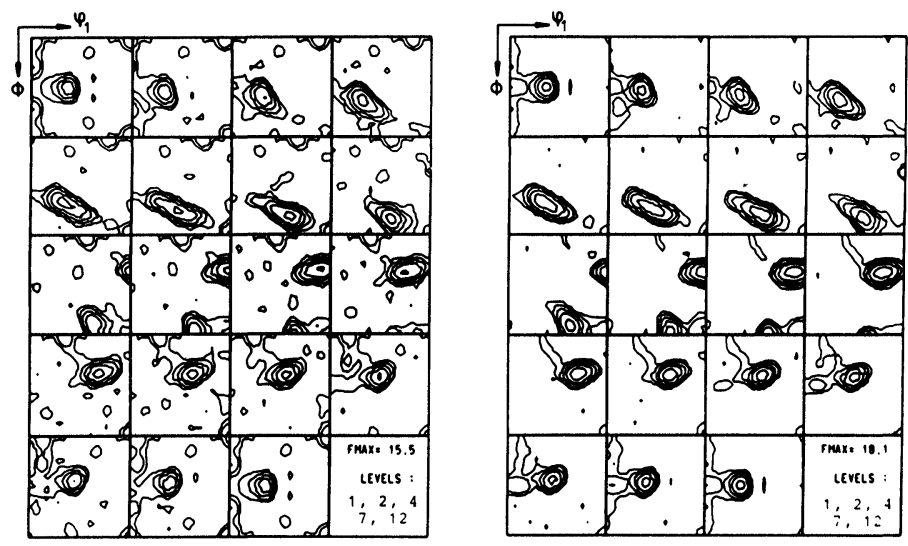

Figure 2 ODFs of (a) $50 \mu \mathrm{m}$ and (b) $500 \mu \mathrm{m}$ grain sized copper after rolling to $95 \%$ and $96 \%$ reduction, respectively. The data was derived from longitudinal sections and mathematically transposed into the standard coordinate system.

rolled specimens. The reason for the remarkable differences between measurements taken from sheet surfaces and longitudinal sections is certainly due to the number of crystals sampled in the two different planes. There are roughly 15 times more crystals in LS compared with the sheet surface, when the initial grain size of $500 \mu \mathrm{m}$ within the applied range of rolling reduction and a penetration depth of the X-rays of $30 \mu \mathrm{m}$ is considered. Furthermore, the number of locations from which measurements are taken and thus the statistical reliability of measurements with increasing strain increases by a factor of 4 from the $93 \%$ to the $98 \%$ rolled specimen, since more strips have to be cut out of the rolling stock.

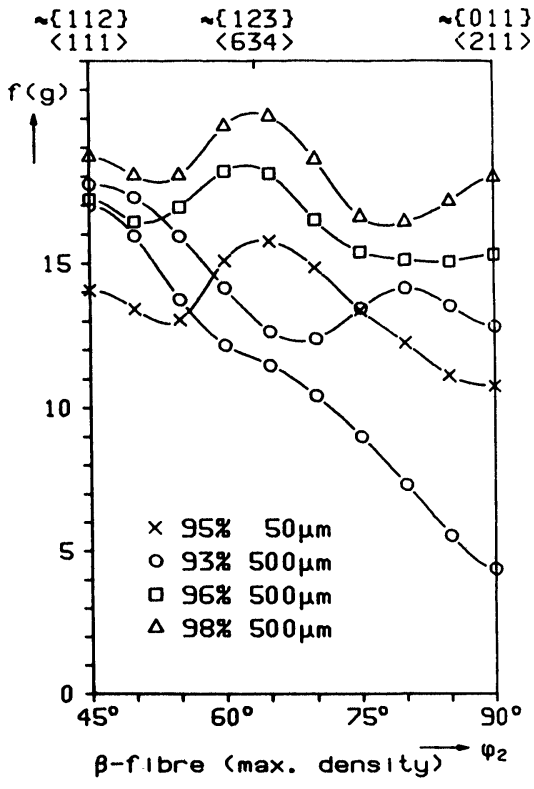

Figure 3 Orientation density along the $\beta$ fibre of the copper textures. The data was derived from longitudinal sections. 
Therefore from hereon, the textures and the data derived from them, are all calculated from LS measurements.

Figure 3 shows the variation of intensity along the $\beta$ fibre between the orientations $\{112\}\langle 111\rangle$ and $\{011\}\langle 211\rangle$, for the $50 \mu \mathrm{m}$ and $500 \mu \mathrm{m}$ grain sized copper. The fine grain sized mateiral after $95 \%$ cold rolling shows the characteristic variations along the $\varphi_{2}=45-90^{\circ}$, and this pattern is followed by the large grain sized material after $96 \%$ and $98 \%$ strain, but at different intensity levels. For $93 \%$ cold rolled copper, however, the statistical sampling is still very poor as can be seen from the two rather different curves obtained from different specimens for this rolling degree (Figure 3).

\subsection{Annealing Textures}

The strongest Cube texture was found in $50 \mu \mathrm{m}$ grain sized copper. Maximum intensity of $\{001\}\langle 100\rangle$ in the ODF deduced from longitudinal section measurements reaches $85 \mathrm{R}$ after rolling $95 \%$ and annealing of $250^{\circ} \mathrm{C}$ (Fig. 4a) which corresponds to the level of $37 \mathrm{R}$ in the (200) pole figure (Figure 5a). For the $500 \mu \mathrm{m}$ grain sized mateiral the ODF for the $96 \%$ rolled specimen is shown in Figure $4 \mathrm{~b}$. In comparison to the texture of the rolled $95 \% 50 \mu \mathrm{m}$ material (Figure 4a) this texture is less sharp and shows besides Cube (28R) a wide orientation spread about the twin of Cube position. The pole figures taken parallel to longitudinal sections in Figures 5b-d show the effect of increasing rolling reduction on the formation of Cube texture; the intensity of Cube increases with strain from less than $4 \mathrm{R}$ after $93 \%$ rolling to $16 \mathrm{R}$ after $98 \%$. The $500 \mu \mathrm{m}$ grain sized copper at the high strain of $98 \%$ (Fig. 5d) produced a weaker texture than the fine grained material after the significantly lower reduction of $95 \%$ (Figure 5a). The shape of the Cube peaks are shown in Figs 6a-c. These are derived from the respective ODFs and exhibit the intensity variation along $\varphi_{1}, \Phi$, and $\varphi_{2}$,
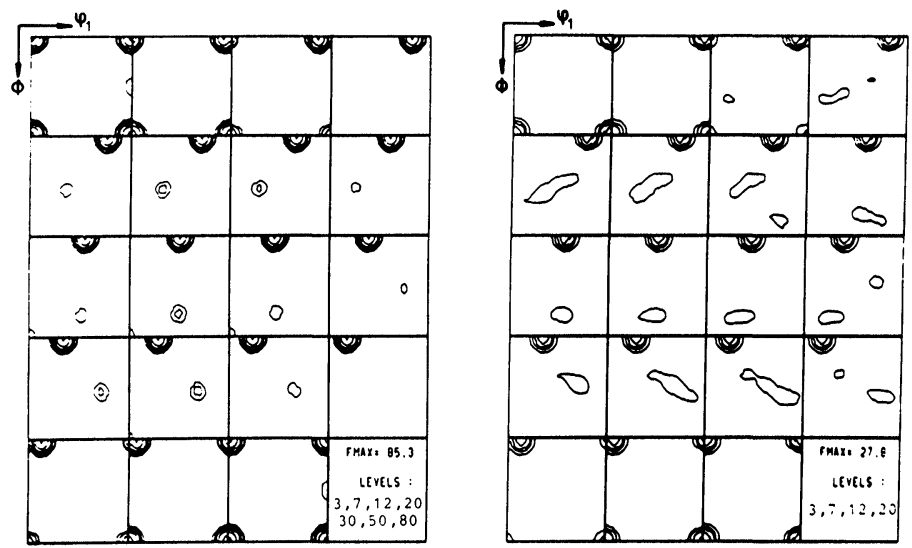

Figure 4 (a) ODF of $50 \mu \mathrm{m}$ grain sized copper after rolling $95 \%$ and full annealing at $250^{\circ} \mathrm{C}$. (b) ODF of $500 \mu \mathrm{m}$ grain sized copper after rolling $96 \%$ and full annealing at $140^{\circ} \mathrm{C}$. The data was derived from longitudinal sections and mathematically transposed into the standard coordinate system. 

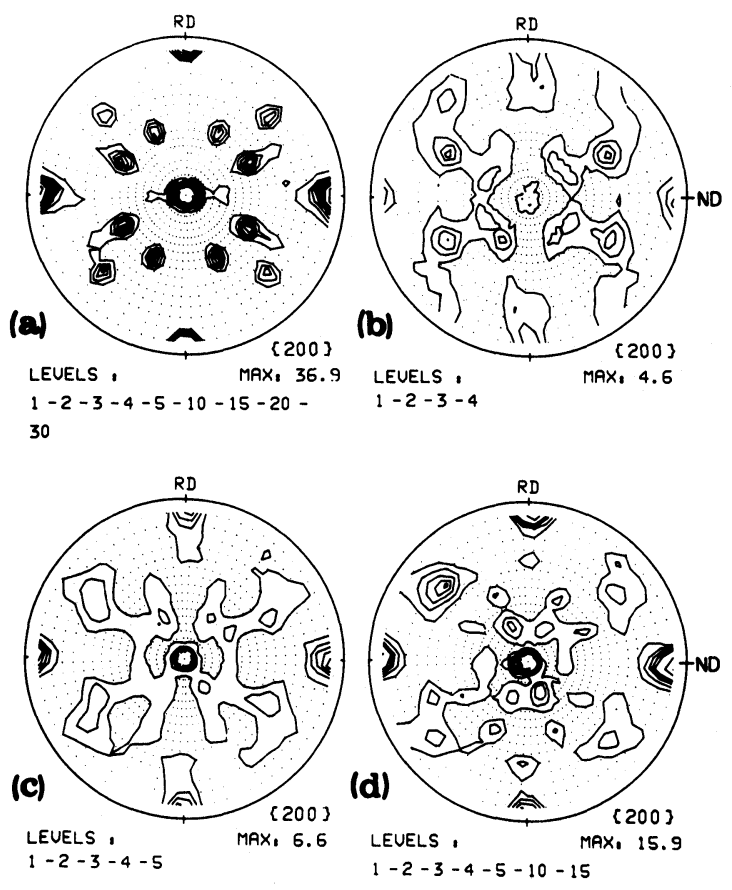

Figure 5 (200) pole figures of fully annealed copper. (a) $50 \mu \mathrm{m}$, cold rolled $95 \%$. (b), (c) and (d) are all from $500 \mu \mathrm{m}$ grain sized material, after rolling $93 \%, 96 \%$, and $98 \%$, respectively. The data was derived from longitudinal sections. (Please note change of axis with respect to the usual way of plotting.)
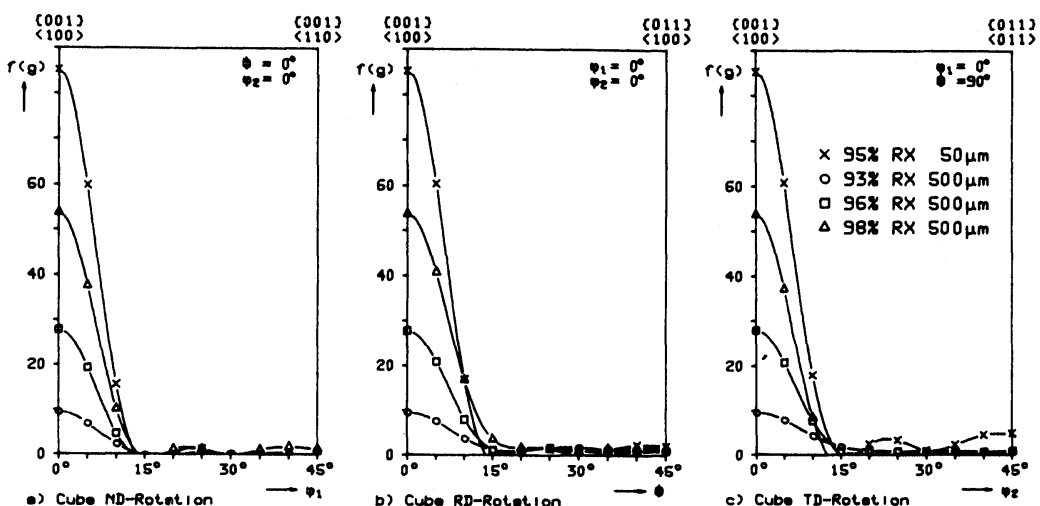

Figure 6 Orientation density of the recrystallized Cube component rotated about (a) ND, (b) RD, and (c) TD. 
which corresponds to $\mathrm{ND}, \mathrm{RD}$, and TD rotations, respectively. There is no evidence of anisotropy in the peak shapes.

Twins of Cube are relatively strong in all cases (Fig. 5) but the ratio of $I_{\text {twin }} / I_{\text {Cube }}$ decreases with rolling strain, reflecting the increasingly dominant Cube component. Careful inspection of Figure 5 shows that the exact positions of the maxima of these twin peaks do not agree with the ideal Cube twin orientation $\{122\}\langle 212\rangle$ and change somewhat with cold rolling. While the deviation from the ideal peak for $500 \mu \mathrm{m}$ copper is about $9^{\circ}$ for $93 \%$ reduction it reduces to $5^{\circ}$ after 98\% reduction. In $50 \mu \mathrm{m}$ copper the deviation from the ideal Cube twin orientation is $2-3^{\circ}$, an angular discrepancy present in many published papers as will be described by Kohlhoff and Lücke in a forthcoming paper (1989).

\section{DISCUSSION}

The fact that in the rolling texture Cube oriented material has been systematically detected in pole figures of longitudinal sections (Fig. 1), but not in those of the sheet surface, supports the notion that these volumes are flat plate-like structures lying parallel to the sheet surface. This is in agreement with earlier TEM studies, by Ridha and Hutchinson (1982) and Hutchinson and Nes (1986). However, it is not possible to measure the way in which Cube intensity in the pole figures varies with strain because the levels are very low. In the fine grain material, which gives the sharpest Cube recyrstallization texture, as well as in the large grain material, the maximum level reached at large strains is about $0.5 \mathrm{R}$. As strain increases from $93 \%$ to $98 \%$, however, there occurs a significant increase in the near-Cube orientations forming the above mentioned fibre. Simultaneously a general sharpening of the components of the copper-type texture was observed.

These changes in the rolling texture coincide with a general strengthening of the Cube recrystallization component (Fig. 5). It is not possible to decide which of these two types of changes in the rolling textures is most important to the formation of Cube recrystallization texture, and indeed it is quite likely that both are essential, since the first affects mainly nucleation and the second growth.

Concerning nucleation, the Dillamore-Katoh model of Cube nucleation envisages Cube oriented subgrains contained in a transition between divergent zones. The effect of increasing strain in this model has two aspects. The first concerns the establishment of sharper curvatures, this favours nucleation. The second aspect deals with the arrival at or near the Cube position of material from increasingly large angular distances. Since the Cube orientation is stable against displacements about $\mathrm{ND}$, but unstable against displacements about $\mathrm{RD}$, material is moving into the Cube orientation by rotating about ND which leads to a narrowing of the pole distribution with respect to ND rotation in the center of the pole figures (Figures $1 b-d$ ). It then rotates further around RD, increasing the range of misorientation of Cube material with respect to $R D$ rotation and thus forming an incomplete $\{h k l\}\langle 100\rangle$ fibre (Figures $1 \mathrm{~b}-\mathrm{d}$ ). However, although the rolling texture results (Figure 1) are consistent with these Dillamore-Katoh predictions of crystal rotations near Cube there remain a number of open questions, if it is assumed that the strength of the Cube component in the recrystallization texture is solely determined by the Cube material in the deformed state. 
(i) As rolling strain increases from $93 \%$ to $98 \%$, Cube texture after recrystallization strengthens although rolling textures do not show a strengthening of the near Cube orientations, something which would be relatively easy to detect. This seems to exclude the idea that the strong Cube texture arises from a higher density of Cube orientated material in the deformed microstructure.

(ii) Surprisingly the Cube recrystallization component is extremely isotropic. The angular range of the Cube recyrstallization component derived from the ODF has for all three axes of rotation the same extension of about $15^{\circ}$, even independent of the rolling degree (Figure 6), although in the deformation texture near Cube material shows a strong spread about Rd. Such a spread, as has been found in many other previous investigations, e.g. by Hirsch and Lücke (1985) should occur in the annealed Cube texture if the DillamoreKatoh mechanism is the major source of Cube nuclei.

These points can partly be explained by the theory of oriented growth. The rolling texture possesses a strong S orientation (Figure 3 ) which is very favourably oriented with respect to growth of Cube. Concerning point (i) the increase of $S$ with strain should lead directly to an increase of Cube, and, moreover, the general sharpening of the rolling texture should lead to a sharpening of the Cube recrystallization texture because growth selection rules become more stringent. Concerning point (ii) the most favourable growth relationship is that between $S$ and the exact Cube position $\left(\approx 40^{\circ}\langle 111\rangle\right)$ so that the nuclei in the distant spread from Cube (by RD rotation) will grow very slowly and thus influence the final texture only little.

There still remains the grain size effect, the origin of which is still a contentious issue. It is obvious that in fine $\mathrm{g}$ rained material the grain-to-grain interactions influence a larger portion of the volume than in coarse-grained material. This interaction should distrub the Taylor-type deformation and then the corresponding deformation texture formation so that the measured texture should vary with grain size (Hansen et al., 1985); but so far no convincing prediction of the texture changes with grain size resulting from such a mechanism has been given. There is also a trivial grain size effect, which is due to insufficient statistics and occurs when the grain size is too large against the measured sample size. This may yield an apparent "grain size" dependence of texture (Duggan and Lee, 1986). In the present investigation, however, this is overcome by choosing longitudinal sections for measurements.

For the weakening of Cube recrystallization textures with increasing initial grain size, only one concrete suggestion has been given. Ridha and Hutchinson (1982) noted that as grain size increases the tendency to shear banding also increases and assumed that these bands destroy the extensive plate-like potential Cube nuclei. The present observation of increasing Cube recrystallization component with rolling from $93 \%$ to $98 \%$ then means that either

(i) the gross rate of Cube nuclei production is faster than the rate at which they are disrupted by shear bands, or

(ii) shear banding becomes less important at higher strains.

Considering (i) there is no evidence that Cube oriented volumes build up significantly as the intensity of this orientation remains at $0.5 \mathrm{R}$ (Figure 1). The second possibility (ii) is also difficult to sustain since the number of shear bands 


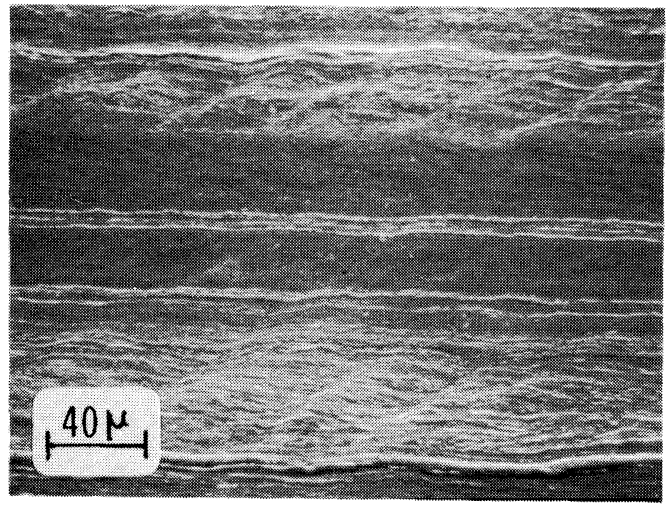

Figure 7 SEM micrograph of etched copper of $500 \mu \mathrm{m}$ grain size after rolling $96 \%$. RD is parallel to the micron marker.

which are part of the deformed microstructure after $96 \%$ reduction (Figure 7) should increase with rolling and thus should prevent Cube recrystallization components from increasing with further rolling. However, this is not observed. The reason for this might be that shear bands as shown in Figure 7 are contained in only certain laminar structures and not in others; that is potential Cube nuclei are not, in general, affected by shear bands, since they are not necessarily contained in volumes prone to shear band formation. Even if shear bands do cut potential Cube nuclei the present investigations does not yield evidence in favour of the assumption that shear band formation is the reason for a lower Cube recrystallization component in large grain sized copper compared to that in fine grain sized copper at similar strains. That the Cube recrystallization component increases with increasing strain underlines however, its strong dependence on the underlying rolling texture which also sharpens with increasing strain rather than on the formation of shear bands, at least within the investigated range of deformation.

\section{CONCLUSIONS}

The accuracy of X-ray back reflection pole figures and ODFs has been imrpoved due to better statistical sampling by using edge sections. Measurements on cold rolled and annealed fine and coarse grain copper has shown that

(i) there is substantial texture development as rolling reduction increases from $93 \%$ to $98 \%$; in particular a low intensity $(<0.5 \mathrm{R})$ fibre of Cube rotated about $\mathrm{RD}$ is formed which is broadly consistent with the Dillamore-Katoh model of deformation in the near Cube volumes of orientation space;

(ii) the shape of Cube oriented deformed material is most probably that of plate-like structures lying parallel to the rolling plane and that the Cube oriented deformed material does not increase with rolling strain over the considered strain range;

(iii) over the strain range considered (93-98\%), the annealing textures show 
very pronounced strengthening of the Cube component for which the Cube oriented plate-like regions can be assumed to act as nuclei;

(iv) it is necessary to invoke the idea of oriented growth to explain the strenghtening of the Cube recrystallization components with increasing strain because Cube does not increase in the deformed state;

(v) initial grain size affects both rolling and annealing textures in so far as larger grain sizes leads to less sharp textures at similar strains and that the increase of the Cube recrystallization component with increasing strain coincides with the sharpening of the respective rolling texture.

\section{Acknowledgements}

The finanical support of the Deutsche Forschungsgemeinschaft for this project is gratefully acknowledged. BJD wishes to thank the DAAD for supporting his visit to Aachen during the summer of 1987. Dipl.-Ing. T. Rickert gave invaluable assistance in the computing work and this is gratefully acknowledged by the authors.

\section{References}

Asbeck, H. O. and Mecking, H. (1978) Mater. Sci. Engng. 34, 111.

Dahl, O. and Pawlek, F. (1936) Z. Metallkde. 28, 266.

Dillamore, I. L. and Roberts, W. T. (1965) Met. Rev. 10, 271.

Dillamore, I. L. and Katoh, H. (1974) Metal. Sci. 8, 73.

Duggan, B. J. and Lee, W. B. (1986) 7th Risø International Symposium on Metallurgy and Materials Science, Roskilde, 297.

Hansen, N., Bay, B., Juul Jensen, D., and Leffers, T., (1985) 7th Conf. on Strength of Metals and Alloys, Montreal, 317.

Hirsch, J. and Lücke, K. (1985) Acta metall. 33, 1927.

Hirsch, J., Burmeister, G. Hoenen, L., and Lücke, K., (1986) In Experimental Techniques of Texture Analysis, ed. H. J. Bunge, DGM-Verlag.

Hirsch, J. and Lücke, K., (1988) Acta metall. 36, 2863.

Hutchinson, W. B. and Nes, E., (1986) 7th Ris $\varnothing$ International Symposium on Metallurgy and Materials Science, Roskilde, 107.

Köhlhoff, G. D. and Lücke, K. (1989), to be published.

Lücke, K., Pospiech, J., Virnich, K. H., and Jura, J., (1981) Acta metall. 29, 167.

Lücke, K., (1984) Proc. 7th Int. Conf. on Textures of Materials, Holland, 195.

Ridha, A. A. and Hutchinson, W. B., (1982) Acta Metall. 30, 1929.

Schmidt, U. and Lücke, K., (1979) Texture of Crystalline Solids, 3, 85.

Wassermann, G., and Grewen, J., (1962) Texturen Metallischer Werkstoffe, Springer-Verlag, Berlin. 